\title{
Rehabilitation of executive function in chronic paediatric brain injury: a randomized controlled trial
}

Anne E. Brandt ${ }^{1,2^{*}}$ (D) Torun G. Finnanger ${ }^{1}$, Ruth E. Hypher ${ }^{3}$, Torstein B. Rø $\varnothing^{1,2}$, Eva Skovlund ${ }^{4}$, Stein Andersson ${ }^{5}$, Kari Risnes ${ }^{1,2+}$ and Jan Stubberud $3,5,6+$

\begin{abstract}
Background: Impaired executive functions (EFs, i.e., purposeful, goal-directed behaviour) cause significant disability after paediatric acquired brain injury (pABI) warranting efficient interventions. Goal Management Training (GMT) is a metacognitive protocol proven effective for executive dysfunction in adults. This pre-registered, blinded, parallelrandomized controlled trial evaluated efficacy of a paediatric adaptation (pGMT) compared to a psychoeducative control (paediatric Brain Health Workshop, pBHW) to improve EF.
\end{abstract}

Methods: Children aged 10 to 17 years with pABI (e.g., traumatic brain injury, brain tumour), $\geq 1$ year post-onset or ended treatment, with parent-reported EF complaints were eligible. Participants were randomized (computeralgorithm) to either group-based pGMT $(n=38)$ or pBHW $(n=38)$. The active control was tailored to keep nonspecific factors constant. Thus, both treatments comprised of 7 sessions at hospitals over 3 consecutive weeks, followed by 4 weeks of telephone counselling of participants, parents, and teachers. Parent-reported daily life EF, assessed by the questionnaire Behavior Rating Inventory of Executive Function (BRIEF; Behavioral Regulation Index (BRI) and Metacognition Index (MI)), were co-primary outcomes 6 months post-intervention. Secondary outcomes included neuropsychological tests and a complex naturalistic task (Children's Cooking Task).

Results: Seventy-three participants (96\%) completed allocated interventions and 71 (93\%) attended the 6-month follow-up. The results demonstrated no significant difference in effectiveness for the two interventions on parentreported EF: For BRIEF $F_{B R I,}$ mean (SD) raw score for pGMT was 42.7 (8.8) and 38.3 (9.3) for pBHW. Estimated difference

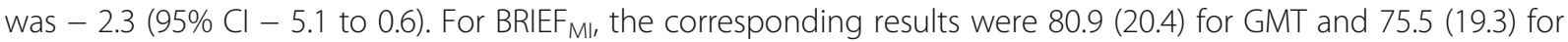
pBHW. Estimated difference was $-1.4(95 \% \mathrm{Cl}-8.5$ to 5.8). In performance-based tests, pGMT was associated with improved inhibition and executive attention, while pBHW was associated with fewer errors in the naturalistic task.

\footnotetext{
* Correspondence: anne.elisabeth.brandt@stolav.no

${ }^{\dagger}$ Kari Risnes and Jan Stubberud contributed equally as co-senior authors.

${ }^{1}$ Children's Clinic, St. Olavs Hospital, Trondheim University Hospital, Postbox

3250 Torgarden, NO-7006 Trondheim, Norway

${ }^{2}$ Department of Clinical and Molecular Medicine, Norwegian University of Science and Technology, Trondheim, Norway

Full list of author information is available at the end of the article
}

(c) The Author(s). 2021 Open Access This article is licensed under a Creative Commons Attribution 4.0 International License, which permits use, sharing, adaptation, distribution and reproduction in any medium or format, as long as you give appropriate credit to the original author(s) and the source, provide a link to the Creative Commons licence, and indicate if changes were made. The images or other third party material in this article are included in the article's Creative Commons licence, unless indicated otherwise in a credit line to the material. If material is not included in the article's Creative Commons licence and your intended use is not permitted by statutory regulation or exceeds the permitted use, you will need to obtain permission directly from the copyright holder. To view a copy of this licence, visit http://creativecommons.org/licenses/by/4.0/ The Creative Commons Public Domain Dedication waiver (http://creativecommons.org/publicdomain/zero/1.0/) applies to the data made available in this article, unless otherwise stated in a credit line to the data. 
Conclusions: In pABI, metacognitive training (pGMT) did not demonstrate additional effectiveness on parentreported daily life EF at 6-month follow-up, when compared to a psychoeducative control. Both interventions were well-tolerated and demonstrated distinct improvements at different EF assessment levels. To conclude on pGMT efficacy, larger studies are needed, including further investigation of appropriate assessment levels and possible differences in effect related to treatment duration, developmental factors, and injury characteristics.

Trial registration: ClinicalTrials.gov, NCT0321534211, 11 July 2017

Keywords: Executive function, Cognition, Paediatric acquired brain injury, Goal management training, Cognitive rehabilitation

\section{Background}

Paediatric acquired brain injuries (pABIs), either traumatic brain injury (TBI) or non-traumatic (e.g., tumour, cerebrovascular accident, infection) are leading causes of childhood morbidity, mortality [1], and acquired disability [2]. As pABI occurs during crucial brain development, the consequences go beyond the immediate brain injury, affecting social competence, behavioural functioning, and cognition [3-5]. Indeed, executive dysfunction represents one of the most common and disturbing cognitive symptoms after pABI. Executive function (EF) refers to cognitive processes responsible for purposeful, goal-directed behaviour [6], operationalized $[7,8]$ in terms of three interrelated core processes: (a) updating (adding relevant and omitting non-relevant information from working memory), (b) shifting (switching between task sets), and (c) inhibition (suppressing or resisting prepotent responses) [7-10]. Executive dysfunction has a substantial global negative impact on everyday life [11-14]. Despite this, there is no consensus regarding cognitive rehabilitation of EF following pABI [15-17].

There is solid empirical support for group-based cognitive interventions for adult ABI [18], with Goal Management Training (GMT) as one of the best validated protocols [19]. The theoretical foundation of GMT holds that the sustained attention system upholds higher-order goals in mind while inhibiting automatic processes [20, 21]. GMT addresses both core (e.g., inhibition and attention) and metacognitive processes (e.g., problem solving), a duality considered especially efficient [22]. Indeed, the effectiveness of GMT has been demonstrated across adult aetiologies, with reports of improved sustained and executive attention [23], assumed essential for daily life EF $[24,25]$ and for global outcomes such as education and independence [12, 26, 27]. Of note, paediatric GMT (pGMT) have been piloted [28] and found to be both feasible and acceptable [29].

The present study addressed previous methodological shortcomings by employing a robust RCT design including blinded assessments, long-term follow-up, active involvement of parents, and counselling of teachers [17,
30-32] as it is imperative to teach and support EF skills in the context of everyday activities and in close cooperation with the adults in the child's life [30, 33]. Most pABI research focuses on single aetiologies [34]. However, as GMT has shown trans-diagnostic effects, the findings suggest that the intervention may be effective across paediatric aetiologies. Due to its multifaceted nature, the assessment of EF is challenging [7, 35-38]. Hence, comparing research employing conventional performance-based tests to studies employing rating scales involves uncertainty, as to whether they index the same underlying EF constructs [39]. Moreover, as GMT targets several EF aspects pertaining to both core processes (bottom-up) and metacognition (top-down), it addresses all levels of functional ability [38]. Thus, assessment should take into account the multifaceted nature of EF, addressing different aspects of EF potentially affected by treatment instead of only addressing one $[35,40]$. Consequently, we used the International Classification of Functioning, Disability and Health (ICF) [41] as the basis to choose the outcome instruments: questionnaire (activity), performance-based neuropsychological tests (impairment) [42], and the novelty of adding a practical task (participation) [43].

The aim of the present study was to examine the efficacy of the metacognitive group-based pGMT, proven efficient in adults, for children in the chronic phase of pABI and with reported daily life executive difficulties. As a comparator, we chose a previously used groupbased active control intervention (psychoeducation; paediatric Brain Health Workshop, pBHW) that was specifically tailored to keep non-specific factors constant (e.g., same therapists, corresponding structure, and duration of training and involvement of parents and teachers). Based on adult studies, we expected greater improvement in executive dysfunction in the pGMT group compared to the pBHW group. The two subindexes of the Behavior Rating Inventory of Executive Function (BRIEF, parent report) were co-primary outcomes. Secondary outcome measures were neuropsychological tests assessing core EF processes [7, 44], as well as a practical complex naturalistic task [45]. 


\section{Methods}

\section{Trial design}

This is an evaluator-blinded, parallel-RCT, with a previously published trial protocol [46], set at two paediatric hospitals in Norway, St. Olavs hospital, Trondheim University Hospital and Oslo University Hospital, Rikshospitalet. The sample size was initially calculated using the Global Executive Composite (GEC) from the BRIEF (parent report) [47], assuming an effect size of .70, significance level of $.05,80 \%$ power, and a $t$-test for difference in means, yielding samples of 32 individuals per group. Later, however, the Behavioral Regulation (BRI) and Metacognition (MI) indexes from BRIEF (that form the GEC), were considered to be more appropriate as co-primary outcomes. For practical and logistical reasons, it was not feasible to recalculate and increase the sample size of the study. Therefore, this study should not be considered as confirmatory as it is likely to be underpowered to find differences between the two groups, if they exist.

Participants were randomized to either pGMT or pBHW in a 1:1 ratio, by blocks of 4 , and stratified by hospital site (Trondheim vs Oslo) and age at intervention (10-13 years vs 14-17 years). A computer-based algorithm was set up for randomization by an independent allocator. The following blinding procedures were applied to reduce systematic bias: (i) Treatment allocation was not disclosed to participants and families, and they were encouraged not to discuss course content outside their group. (ii) "Brain training" was used consistently in both groups. (iii) Independent, trained, and blinded test technicians conducted assessments. (iv) Group therapists were blinded to all assessments. (v) Blinded data monitoring and statistical analysis were implemented. Finally, interventions were arranged in random order.

Written informed consent was provided from primary caregivers of participants ( $<16$ years) and from participants and their caregivers ( $>16$ years). Study procedures and monitoring according to Norwegian Clinical Studies Infrastructure Network procedures.

\section{Recruitment and eligibility}

Participants eligible for a written invitation were identified based on discharge diagnosis and medical records (Fig. 1) [46]. Further eligibility screening was done in an initial telephone contact and more thoroughly in a semistructured screening interview [see Additional file 1] with primary caregivers (or participants surpassing 16 years). Successively, after written informed consent, eligible individuals were designated a study number and randomized.

Eligible children were aged 10 to 17 years, diagnosed with pABI (TBI, brain tumour, cerebrovascular accident, hypoxia/anoxia, or brain infection/inflammation), at intervention $>12$ months post-insult or $>12$ months stable brain tumour situation/ended cancer therapy, with reported executive function difficulties in daily life as described in the screening interview by parents (or participants). Exclusion criteria included pABI before the age of 2 years; cognitive (including memory), physical, or language impairments affecting the capacity to follow educational goals of peers and attend regular classroom teaching; pre-injury neurological disease; severe psychiatric disorder and/or on stimulant medication; recently detected brain tumour relapse; or not fluent in Norwegian. To our knowledge, none of the participants had prior to the study received specific cognitive rehabilitation. Recruitment lasted from November 2017 until May 2019 and was ended at achieved sample size.

\section{Interventions}

The two group-based interventions compared in this study were the metacognitive pGMT and an active control (pBHW) involving the use of educational materials and lifestyle topics, similar to many other psychoeducational ABI rehabilitation programmes [48]. While the pBHW programme was not specifically aimed at remediating EF, the $\mathrm{pBHW}$ programme was tailored to keep non-specific factors constant (i.e., structure and intensity/duration of training, between session-assignments, involvement of parents and teachers, professional attention, group dynamics).

\section{Paediatric goal management training}

The manualized $\mathrm{GMT}^{\oplus}$, adult protocol has previously been translated into Norwegian [49, 50]. A preliminary paediatric protocol was developed and piloted by our research group [29] and then further refined based on the feedback for the present study. The pGMT promotes inhibitory control and metacognitive strategies and facilitation of goal-achievements, in addition to mindfulness training [51]. The protocol consists of 7 sessions, each of 2-h duration (Fig. 2). Original GMT materials were made more ageappropriate (e.g., examples and discussions more related to school and leisure activities, children portrayed in illustrations instead of adults, language simplified, more visual materials, and movie clips were included).

\section{Active control intervention}

The original adult Brain Health Workshop (BHW) protocol is a manualized psychoeducational intervention developed to match GMT for non-specific factors $[20,24]$, and adapted into a paediatric version in a similar manner as described for pGMT. pBHW 


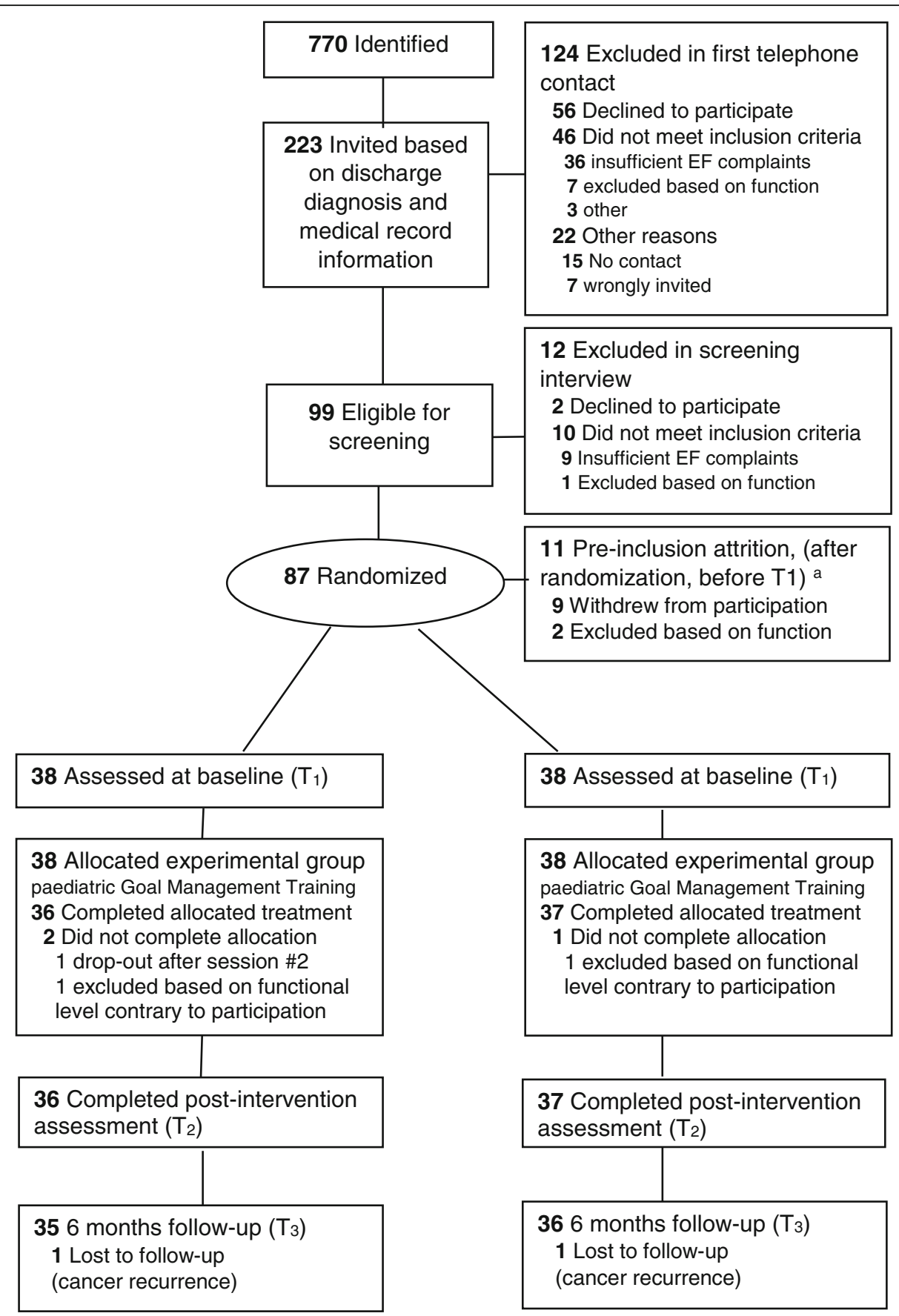

Fig. 1 Consort flow diagram. ${ }^{a}$ Nine withdrew while waiting for start of assigned intervention group (worsening of illness, medication testing, intensifying physical rehabilitation), 2 were excluded post-randomization (before baseline) after identification of violations of eligibility criteria not previously communicated. Attrition distribution was evenly distributed between the two groups, 6 in pGMT and 5 in pBHW. See also Additional file 4, Consort checklist

represents an active control intervention including educational materials addressing brain injury and (dys)function, plasticity, memory and learning, EF, fatigue, and lifestyle issues (e.g., stress, sleep, exercise and nutrition) [20, 24] (Fig. 2).

\section{Similarities of treatments for non-specific factors and treatment fidelity}

The two group-based interventions were corresponding regarding structure, intensity, duration, therapist contact, and access to distributed materials. Treatment 


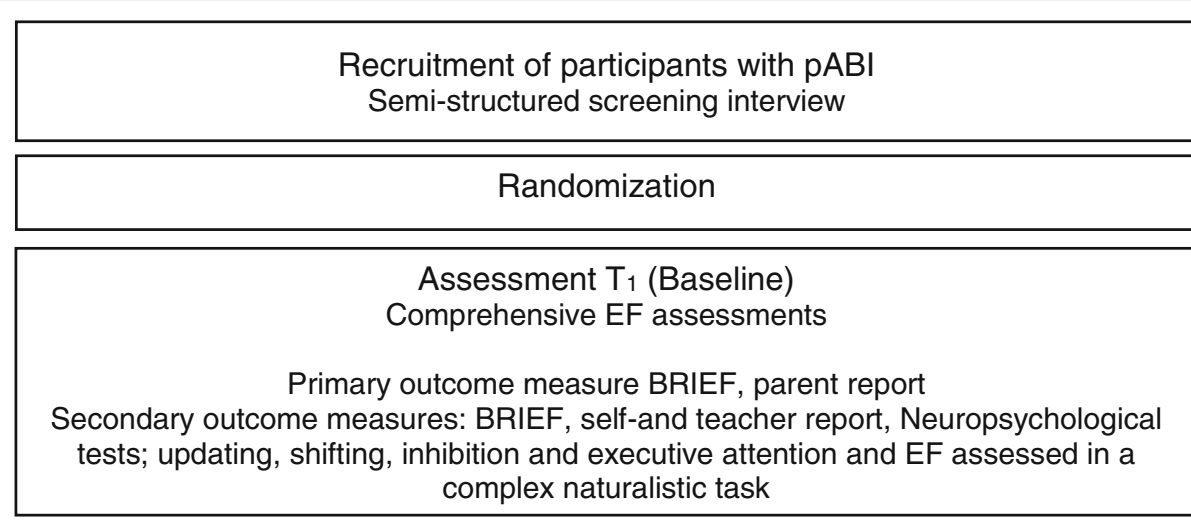

Paediatric Goal Management
Training (pGMT, $n=38$ )
Metacognitive intervention:
Modul 1: The absent mind, the present mind
Modul 2: Absentminded slip-ups
Modul 3: The automatic pilot, "stop"
Modul 4: The brain blackboard (i.e. working
memory)
Modul 5: State your goal
Modul 6: Splitting tasks into subtasks
Modul 7: Checking ("Stop")

Hospital sessions (2 hours) of 7
modules over 3 subsequent weeks.
Groups of 2-5 participants, ideally 4, led
by two experienced neuropsychologists.
Manualized with PowerPoints.
Between session assignments.
External cuing by key word "Stop".
Training and education of parents.
Paediatric Brain Health Workshop (pBHW, $n=38$ )

Psychoeducation intervention:

Modul 1: Introduction, pABI

Modul 2: CNS and neuroplasticity

Modul 3: Memory I

Modul 4: Memory II

Modul 5: EF and attention

Modul 6: Lifestyle and neuroplasticity I, incl.

stress, sleep and energy

management/fatigue.

Modul 7: Lifestyle and neuroplasticity II, incl. physical exercise and nutrition.

Hospital sessions (2 hours) of 7

modules over 3 subsequent weeks.

Groups of 2-5 participants, ideally 4 , led by two experienced neuropsychologists. Manualized with PowerPoints.

Between session assignments.

External cuing by key word "Brain

training".

Training and education of parents.

\section{External cuing period and telephone-counselling (5 weeks)}

Following the fourth session, external cuing (i.e., $18 \mathrm{SMS}$ );

Two hours (2x1) telephone counselling for parents/participants. One-hour counseling given teacher in the same period for each participant.

Assessment $\mathrm{T}_{2}$ (immediately post-intervention at 8 weeks)

Assessment $T_{3}$ (6-months follow-up)

Fig. 2 Outline of the study design. PABI, paediatric acquired brain injury; EF, executive function; BRIEF, Behavior Rating Inventory of Executive Function; pGMT, paediatric Goal Management Training; pBHW, paediatric Brain Health Workshop

fidelity was ensured by two trained therapists at each site and standardization of materials to warrant consistent and reliable implementation. Accompanying all sessions, pre-prepared manualized PowerPoint slides were used to standardize content across groups and sites. Additionally, participant workbook inserts specific for each session were handed out at the start of each session. Both interventions included discussions of participants' real-life experiences and attention deficits, in-session practice, between-sessions exercises, monitoring of activity, education of parents and teachers, and periodic alertness cueing following the fourth session (SMS; stating 
the key term "STOP" in pGMT and the more general "Brain training" in pBHW) to facilitate transfer to daily living [52, 53]. If a participant was prevented from attending a group session, an individual session of the missed module was offered before the following to ensure progress. Parents received a 1-h review after every paediatric session with group therapists allowing questions and exchange of experience. In the 4 weeks following sessions, telephone counselling was conducted to promote use of the interventions. All designated teachers received written information about the study and a 1-h telephone counselling post sessions that included a brief review and encouragement to implement intervention principles [30]. Last follow-up was conducted in November 2019.

\section{Baseline assessment}

Medical characteristics at the time of insult were extracted from the medical records (Table 1). At start of intervention $\left(T_{1}\right)$, systematic assessments of baseline sociodemographic factors and medical history as well as a standardized physical examination was performed for all individuals by a study nurse and a paediatrician. Fatigue was measured with the Pediatric Quality of Life Inventory-Multidimensional Fatigue Scale (PedsQL MFS, parent report) [54]. Parents rated each item according to their child's function the prior month (e.g., "Feels too tired to spend time with his/her friends"). Presented are reversed total score, linearly transformed to a 0-100 scale. A higher score indicates fewer fatigue symptoms. General intellectual ability was measured by full scale intelligence quotient (FSIQ) from Wechsler Intelligence Scale for Children-Fifth edition (WISC-V), with normalized IQ distribution $(M=100, \mathrm{SD}=15)$. Blinded baseline assessment was performed the day prior to initiation of the interventions as several participants had a long commute to the research site and reported fatigue, thus avoiding the burden of an additional travel just for the assessment.

\section{Outcome measures}

An accurate and valid evaluation of EF benefits from assessments at different levels to obtain clinically useful functional ability information $[38,55]$. Since pGMT addresses all three ICF- levels [41], EF outcome measures pertaining the three levels were included by questionnaire (activity level, primary outcome measure), performance-based tests (impairment level, secondary outcome measure), and a complex naturalistic task (participation level, secondary outcome measure) [38, 43, 56]. Investigators blinded to the intervention performed assessments at pre-intervention (baseline, $T_{1}$ ), postintervention $\left(T_{2}\right)$, and at 6-month follow-up $\left(T_{3}\right)$.
The BRIEF parent report [47] at $T_{3}$ was employed as the primary EF outcome measures using the two indexes, the BRI and the MI as co-primary endpoints (raw scores BRIEF $_{\mathrm{BRI}}$ and $\mathrm{BRIEF}_{\mathrm{MI}}$ ). Since the BRIEF has been used as a primary outcome in adult GMT studies [49] results can potentially be compared. The BRIEF is an 86-item standardized questionnaire designed to capture parent perceptions of a child's everyday EF. Each item's frequency of occurrence is rated on a 3-point Likert scale from 1 (never) to 3 (often), higher scores indicate greater dysfunctions. The BRIEF has proven applicable to several clinical groups [57] and has demonstrated adequate internal consistency, inter-rater and test-retest reliability $[47,58]$ for parent report, in addition to selfand teacher reports (secondary outcomes).

Secondary outcome assessments of EF at the impairment level were conducted with standardized neuropsychological tests pertaining updating (digit span, total score, raw score (Wechsler Intelligence Scale for Children-Fifth edition, WISC-V)) [59]; shifting (Trail Making Test 4 (TMT 4); total time; raw scores from the Delis-Kaplan Executive Function System (D-KEFS) [60]; inhibition (Conners' Continuous Performance Test, 3ed (CPT-III) Commissions, $T$-scores) [61]; and executive attention (Color Word Interference Test 4 (CWIT 4, DKEFS), total time, raw scores) [60]. All tests are paper and pencil tests, except CPT-III, which is a computerized assessment of different aspects of attention. Higher test scores on CPT-III and digit span indicated better performance, whereas higher scores on TMT 4 and CWIT 4 indicated poorer performance.

Finally, at the participation level, we included a complex open-ended task, the Children's Cooking Task (CCT), total errors, and raw scores [45, 62]. The task (cooking) required multitasking in a naturalistic setting (kitchen) with aspects of novelty and distractions and, hence, less external control and structure. The CCT has demonstrated sensitivity to executive dysfunction in pABI [63].

\section{Statistical analysis}

Categorical data were summarized by counts and percentages. Continuous data were summarized by mean and standard deviation (SD) or median and interquartile range (IQR), presented for each group. Any baseline differences between the groups were viewed as incidental [64] due to the randomization, but noted. A statistical analysis plan (SAP) was outlined in advance and registered at ClinicalTrials.gov prior to un-blinding of the data. The primary analysis was carried out blind of allocation by a statistician. The within and between differences of the two co-primary endpoints constituting BRIEF (raw scores BRIEF $\mathrm{BRI}_{\text {and }}$ aRIEF $\mathrm{MI}_{\mathrm{MI}}$ ) were estimated by linear mixed models [65] in a full analysis set 
Table 1 Participant demographics, injury characteristics and cognitive scores at baseline $\left(T_{1}\right)$

\begin{tabular}{|c|c|c|c|}
\hline & \multicolumn{2}{|l|}{ Interventions } & \multirow{2}{*}{$\begin{array}{l}\text { Total }(n= \\
76)\end{array}$} \\
\hline & pGMT $(n=38)$ & pBHW $(n=38)$ & \\
\hline \multicolumn{4}{|l|}{ Demographics } \\
\hline Sex, girls, No. (\%) & $21(55)$ & $22(58)$ & $43(57)$ \\
\hline \multicolumn{4}{|l|}{ Maternal educational level, №. $(\%)^{\text {a }}$} \\
\hline Primary school & $2(5)$ & $2(5)$ & $4(5)$ \\
\hline High school & $9(24)$ & $14(37)$ & $23(30)$ \\
\hline University/college & $25(66)$ & $21(55)$ & $46(61)$ \\
\hline Intact family unit, No. (\%) & $24(63)$ & $26(68)$ & $50(66)$ \\
\hline \multicolumn{4}{|l|}{ Age at intervention, No. (\%) } \\
\hline $10-13$ years & $20(53)$ & $20(53)$ & $40(53)$ \\
\hline $14-17$ years & $18(47)$ & $18(47)$ & $36(47)$ \\
\hline \multicolumn{4}{|l|}{ Hospital site, No. (\%) } \\
\hline St. Olavs Hospital Trondheim & $20(53)$ & $22(58)$ & $42(55)$ \\
\hline Oslo University Hospital & $18(47)$ & $16(42)$ & $34(45)$ \\
\hline \multicolumn{4}{|l|}{ Injury characteristics } \\
\hline Age at injury, median (IQR), years & $8.4(5.3-10.9)$ & $8.5(6.3-11.1)$ & $8.5(5.9-11)$ \\
\hline Time since injury, median (IQR), years & $4.8(2.8-6.6)$ & $5.5(3.3-7.5)$ & $5.3(3.3-7.3)$ \\
\hline \multicolumn{4}{|l|}{ Primary injury, №. (\%) } \\
\hline Brain tumour & $15(40)$ & $14(37)$ & $29(38)$ \\
\hline Traumatic brain injury & $7(18)$ & $11(29)$ & $18(24)$ \\
\hline Cerebrovascular accidents & $10(26)$ & $7(18)$ & $17(22)$ \\
\hline Infection/Inflammation & $3(8)$ & $4(11)$ & $7(9)$ \\
\hline Hypoxia/Anoxia & $3(8)$ & $2(5)$ & $5(7)$ \\
\hline Admitted to intensive care unit, №. (\%) & $25(66)$ & $24(65)$ & $49(65)$ \\
\hline No. of days in intensive care unit, median (IQR) & $4.0(1.0-7.0)$ & $2.0(1.0-7.0)$ & $2.0(1.0-7.0)$ \\
\hline Cerebral imaging, No. (\%) ${ }^{\mathrm{b}}$ & $38(100)$ & $38(100)$ & $76(100)$ \\
\hline Confirmatory findings, No. (\%) ${ }^{\mathrm{c}}$ & $36(95)$ & $31(82)$ & $67(88)$ \\
\hline Pathological neurological findings at baseline, No. (\%) & $20(53)$ & $13(34)$ & $33(43)$ \\
\hline Fatigue at baseline, reversed total score & $54.7(19.3)$ & $55.9(19.3)$ & $55.3(19.2)$ \\
\hline \multicolumn{4}{|l|}{ Cognitive scores ${ }^{d}$, mean (SD) } \\
\hline Intellectual ability (FSIQ), age normed $(n=72)$ & $92.5(13.2)$ & $92.4(13.6)$ & $92.5(13.3)$ \\
\hline BRIEF $_{\text {BRI }}$ parent, $T$-score $(n=76)$ & $57.2(9.4)$ & $55.4(15.1)$ & $56.3(12.5)$ \\
\hline BRIEF $_{\text {MI }}$ parent, $T$-score $(n=75)$ & $62(9.6)$ & $60.1(11.3)$ & $61.1(10.4)$ \\
\hline 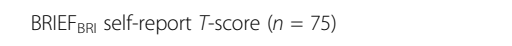 & $50.9(11.7)$ & $52.1(12.1)$ & $51.5(11.8)$ \\
\hline BRIEF $_{\text {MI }}$ self-report, $T$-score $(n=75)$ & $55.7(12.7)$ & $57.2(12.7)$ & $56.4(12.6)$ \\
\hline BRIEF $_{\text {BRI }}$ teacher report, $T$-score $(n=72)$ & $57.6(15.9)$ & $59.3(18.0)$ & $58.5(16.9)$ \\
\hline BRIEF $_{\text {MI }}$ teacher report, $T$-score $(n=71)$ & $61.5(11.8)$ & $61.5(16.5)$ & $61.5(14.3)$ \\
\hline Updating, scaled score $(n=73)$ & $9.3(2.9)$ & $8.5(2.8)$ & $8.9(2.8)$ \\
\hline Shifting, scaled score $(n=76)$ & $8.5(3.9)$ & $7.8(3.5)$ & $8.1(3.7)$ \\
\hline Inhibition, T-score $(n=76)$ & $51.3(8.6)$ & $54(7.7)$ & $52.6(8.3)$ \\
\hline Executive attention, scaled score $(n=76)$ & $7.4(3.9)$ & $8.0(3.2)$ & $7.7(3.6)$ \\
\hline Complex naturalistic task, total errors $(n=72)$ & $27.4(19.6)$ & $29(23.7)$ & $28.3(21.7)$ \\
\hline
\end{tabular}

pGMT paediatric Goal Management Training, pBHW paediatric Brain Health Workshop, IQR Interquartile range, BRIEF Behavior Rating Inventory of Executive Function, BRI Behavioral Regulation Index, MI Metacognition Index

a Only 73 out of 76 mothers $(96 \%)$ stated their level of education

${ }^{\mathrm{b}}$ All participants had conducted magnetic resonance imaging (MRI) or computed tomography (CT) at some point. Of these, 4 individuals (11\%) in the pGMT group and 7 (18\%) in

the pBHW group had only performed CT

c Out of the 9 individuals without confirmatory imaging (normal), 6 had sustained TBI, 1 hypoxia, and 2 with brain infection/inflammation. Three out of 9 participants without confirmatory imaging had conducted a CT only and all of these had TBI. ${ }^{d}$ Fatigue is presented as reversed total score, linearly transformed to a $0-100$ scale. A higher score indicate fewer fatigue symptoms

e Specifications cognitive measures

Intellectual ability: Full Scale Intelligence Quotient $(F S I Q)$ from Wechsler Intelligence Scale for Children-Fifth edition (WISC-V) with normalized IQ distribution $(M=100$, SD = 15). Only

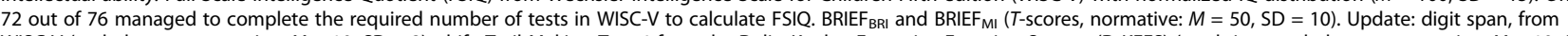

WISC-V (scaled score, normative: $M=10, S D=3$ ); shift: Trail Making Test 4 from the Delis-Kaplan Executive Function System (D-KEFS) (total time, scaled score, normative: $M=10, S D$ = 3); inhibition: commissions from Conners' Continuous Performance Test, 3ed (CPT-III) ( $T$-scores, normative: $M=50, S D=10)$; and executive attention: Color Word Interference Test 4 (CWIT4, D-KEFS) (total time, scaled score, normative: $M=10, \mathrm{SD}=3$ ). Complex naturalistic task: Children's Cooking Task (CCT) (raw score, total errors) 
(FAS). All randomized individuals with post-baseline outcome data (ICH E9) [66] were included, representing an intention-to-treat approach. The model included baseline raw scores as a covariate [67], and treatment group, time (post-treatment $\left(T_{2}\right)$ and at 6 months follow-up $\left(T_{3}\right)$ ), interaction between time and treatment, and randomization strata (1) site (Trondheim or Oslo) and (2) age at intervention (10-13 or 14-17 years) as fixed factors. Time was included as categorical with unstructured covariance as the starting point. According to the SAP, the per-protocol population included participants who had missed a maximum of 2 out of the 7 group sessions. Since all participants who completed the allocated intervention $(n=73)$ underwent all 7 modules the main analysis (FAS) and per-protocol were identical. The two co-primary endpoints from BRIEF (BRI and ${ }_{\text {MI }}$ ) were analysed using the Hochberg procedure to control for the type I error rate [68]. The global null hypothesis of no difference between groups was rejected if the test of either endpoint was statistically significant at the twosided 0.025 level, or if both tests were significant at the two-sided 0.05 level. Differences between treatment groups were accordingly estimated with 95\% confidence intervals. Exploratory subgroup analyses of the primary outcome measures were performed according to strata and sensitivity analyses were performed by exploring the effect of additional covariates thought to be of prognostic importance [69]: type of injury (aetiology), age at injury (years), time since injury (years), and reported fatigue at intervention. Secondary outcomes of self and teacher reports (raw scores BRIEF $\mathrm{BRI}_{\mathrm{BR}}$ and $\mathrm{BRIEF}_{\mathrm{MI}}$ ) were also analysed by the linear mixed model. Secondary outcomes pertaining performance-based neuropsychological tests at 6-month follow-up were analysed by linear regression, with baseline raw scores, treatment group, and strata as covariates. For secondary endpoints, the number of outcomes were theory-driven, pre-planned and limited (only one outcome per function) to avoid an inflation of type I error. Estimates of treatment differences were presented with 95\% confidence intervals, and tests were performed using a two-sided significance level of 0.05 . No adjustment of multiplicity was planned. Distributional assumptions were checked by visual inspection of residual plots. No imputation of missing scores was made. Main analysis was conducted March 2020 using SAS v9.4. IBM-SPSS version 26 and Stata16 were used for secondary analyses.

\section{Results}

Flow chart on recruitment is presented in Fig. 1. Of the 99 individuals who were screened by interview, two declined to participate and ten did not meet the eligibility criteria: nine reported insufficient EF complaints and one was excluded for not meeting inclusion criteria for functional level. Thus, 87 were randomized. Since the interventions were group-based (assembled by age, sites, and treatment allocation), some participants experienced a latency from randomization pending initiation of a group. Nine potential participants withdrew from participation before baseline assessment while waiting start of assigned intervention group due to unforeseen changes in life situation (worsening of illness, medication testing, intensifying of physical therapy). Two were excluded post-randomization (before baseline) after identification of violations of eligibility criteria not previously communicated. Pre-inclusion attrition was evenly distributed between the two groups, 6 in pGMT and 5 in pBHW. Demographic, injury characteristics, and cognitive scores at baseline for the 76 participants are summarized in Table 1.

\section{Treatment compliance}

Seventy-three participants (96\%) completed the allocated intervention (Fig. 1). There was no attrition postintervention, and only two participants (one in each group) failed to attend at the 6-month follow-up due to cancer recurrence, resulting in $93 \%$ attendance.

\section{Primary outcomes}

The results show no significant difference between the two intervention groups for the primary outcome (changes in parent-reported BRIEF raw scores from baseline $\left(T_{1}\right)$ to 6 months follow-up $\left.\left(T_{3}\right)\right)$. Thus, estimated between-group differences in the linear mixed model for $\mathrm{BRIEF}_{\mathrm{BRI}}$ was $-2.3(95 \% \mathrm{CI}-5.1$ to 0.6$), p=$ .12 , and the corresponding result for BRIEF $\mathrm{MI}$ was -1.4 (95\% CI -8.5 to 5.8), $p=.71$ (Fig. 3). Exploratory stratified subgroup analyses and sensitivity analyses did not differ meaningfully from the main result [see Additional file 2, 3]. Although no difference in effect for the two interventions was demonstrated, the results show significant decrease in parent-reported executive dysfunction for both interventions. In linear mixed models, the estimated changes from baseline $\left(T_{1}\right)$ to 6-month follow-up $\left(T_{3}\right)$ with $95 \%$ CIs and $\mathrm{p}$ values for $\mathrm{BRIEF}_{\mathrm{BRI}}$ were -3.8 $(-5.6,-1.9, p<.001)$ for pGMT and $-5.7(-8.5,-2.9$, $p<.01)$ for $\mathrm{pBHW}$. The corresponding results for BRIEF $_{\mathrm{MI}}$ were $-6.9(-10.9,-2.9, p=.001)$ for pGMT and $-8.9(-13.2,-3.9, p=.001)$ for $\mathrm{pBHW}$.

\section{Secondary outcomes}

As presented in Fig. 3, comparing pGMT and pBHW at $T_{3}$ mixed modelling revealed no difference in either selfor teacher reports for $\mathrm{BRIEF}_{\mathrm{BRI}}$ or $\mathrm{BRIEF}_{\mathrm{MI}}$, which supports the primary results of no difference between interventions.

Results from performance-based tests and the complex naturalistic task are presented in Table 2 and 


\begin{tabular}{|c|c|c|c|}
\hline Difference between means $(95 \% \mathrm{Cl})$ & Estimate (\%, Cl lower-upper) & Favors pBHW & Favors pGMT \\
\hline \multicolumn{4}{|l|}{ Behavioral Regulation Index } \\
\hline \multicolumn{4}{|l|}{ Parent-reported (FAS analysis) } \\
\hline 8 weeks & $-2.38(-5.38-0.62)$ & & - \\
\hline 6 months & $-2.26(-5.14-0.63)$ & . & - \\
\hline \multicolumn{4}{|l|}{ Teacher-reported } \\
\hline 8 weeks & $-0.45(-3.10-2.20)$ & 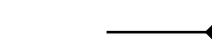 & \\
\hline 6 months & $-0.91(-5.59-3.76)$ & & \\
\hline \multicolumn{4}{|l|}{ Self-reported } \\
\hline 8 weeks & $2.54(-1.69-6.77)$ & & \\
\hline 6 months & $-1.08(-6.32-4.16)$ & & \\
\hline \multicolumn{4}{|l|}{ Metacognition Index } \\
\hline \multicolumn{4}{|l|}{ Parent-reported (FAS analysis) } \\
\hline 8 weeks & $-1.29(-6.73-4.14)$ & & \\
\hline 6 months & $-1.35(-8.52-5.81)$ & & \\
\hline \multicolumn{4}{|l|}{ Teacher-reported } \\
\hline 8 weeks & $1.89(-4.00-7.77)$ & & \\
\hline 6 months & $-0.06(-7.27-7.16)$ & & \\
\hline \multicolumn{4}{|l|}{ Self-reported } \\
\hline 8 weeks & $2.56(-2.66-7.79)$ & & \\
\hline \multirow[t]{3}{*}{6 months } & $-0.63(-6.51-5.26)$ & & \\
\hline & $\Gamma_{-10}$ & $T_{-5}^{T}$ & $\begin{array}{l}1 \\
5\end{array}$ \\
\hline & \multicolumn{3}{|c|}{ Difference between means $(95 \% \mathrm{Cl})$} \\
\hline \multicolumn{4}{|c|}{$\begin{array}{l}\text { Fig. } 3 \text { Estimated differences between treatment groups on the BRIEF questionnaire with 95\% confidence intervals. BRIEF, Behavior Rating } \\
\text { Inventory of Executive Function; pGMT, paediatric Goal Management Training; PBHW, paediatric Brain Health Workshop; FAS, full analysis set. } \\
\text { Estimated differences (pGMT-pBHW) in mean raw scores, adjusted for baseline scores and strata; age at intervention (10 to } 13 \text { years/14 to } 17 \\
\text { years); and study site (Trondheim/Oslo) }\end{array}$} \\
\hline
\end{tabular}

Table 2 Estimated difference between pGMT and pBHW in performance-based tests at 6-month follow-up with 95\% confidence interval

\begin{tabular}{|c|c|c|c|c|}
\hline EF outcomes & pGMT, mean (SD) ${ }^{\mathrm{a}}$ & pBHW, mean (SD) ${ }^{\mathrm{a}}$ & Estimated mean difference $(95 \% \mathrm{Cl})^{\mathrm{b}}$ & $P$ value \\
\hline Updating, raw scores $(n=70)$ & $26.2(5.5)$ & $25.3(4.4)$ & $-0.2(-1.7$ to 1.4$)$ & .830 \\
\hline Shifting, raw scores $(n=70)$ & $94.3(57.6)$ & $96.4(46.2)$ & -4.5 (- 18.9 to 9.9$)$ & .540 \\
\hline Inhibition, T-scores $(n=71)$ & $48.3(9.3)$ & $54.8(9.6)$ & $5.2(1.6$ to 8.7$)$ & .005 \\
\hline Executive attention, raw scores $(n=71)$ & $72.4(31.7)$ & $75.1(22)$ & $9.9(2.2$ to 17.6$)$ & .010 \\
\hline Complex naturalistic task, raw scores $(n=68)$ & $18.7(16.4)$ & $15.8(13.1)$ & $-4.3(-8.4$ to -0.3$)$ & .040 \\
\hline
\end{tabular}

pGMT paediatric Goal Management Training, $p B H W$ paediatric Brain Health Workshop

${ }^{a}$ Unadjusted mean (SD)

${ }^{\mathrm{b}}$ Estimated mean differences from multiple linear regressions including the baseline measure as a covariate. Mean difference in raw scores except for inhibition ( $T$-score), adjusted for baseline scores and strata; age at intervention (10 to 13 years/14 to 17 years) and study site (Trondheim/Oslo). Updating: Digit span, Wechsler Intelligence Scale for Children-Fifth edition (WISC-V) (raw score); shifting: Trail Making Test 4 (TMT 4, from the Delis-Kaplan Executive Function System, D-KEFS) (raw score, total time); inhibition: commissions from Conners' Continuous Performance Test, 3ed (CPT-III) (T-scores, normative: $M=50, S D=10)$; executive attention: Color Word Interference Test 4 (CWIT4, D-KEFS) (raw score, total time); and complex naturalistic task: Children's Cooking Task (CCT) (raw score, total errors) 
demonstrates that at 6-month follow-up, the pGMT group had improved inhibition scores (difference in $T$ score at $T_{3}, 5.2$ (95\% CI 1.6 to 8.7$\left.) ; p=.005\right)$ and executive attention (difference in raw score at $T_{3}, 9.9$ (95\% CI 2.2 to 17.6 ); $p=.01$ ), but not updating (difference in raw score at $T_{3},-0.2(95 \% \mathrm{CI}-1.7$ to 1.4$\left.) ; p=.83\right)$ or shifting (difference in raw score at $T_{3},-4.5(95 \% \mathrm{CI}-18.9$ to 9.9$), p=.54$ ). The $\mathrm{pBHW}$ enhanced performance on the complex naturalistic task (CCT) (difference in raw score at $T_{3},-4.3(95 \% \mathrm{CI}-8.4$ to -0.3$\left.), p=.04\right)$.

\section{Adverse events}

One psychological reaction was reported in the pGMT group at the end of intervention. It was assessed and handled according to study protocol and good clinical practice [70]. Although symptoms originated before study inclusion, it was recorded as an adverse event (moderate) with uncertain connection to the intervention.

\section{Discussion}

The aim of the present study was to examine the efficacy of pGMT, adapted from a metacognitive protocol proven effective in adults, for children in the chronic phase of pABI with EF complaints, when compared to psychoeducation ( $\mathrm{pBHW}$ ). Keeping non-specific factors constant, the metacognitive intervention did not demonstrate an additional effect in reducing daily life executive dysfunction. Secondary, performance-based tests, however, showed more improvement in pGMT pertaining inhibition and executive attention, while pBHW demonstrated better performance in the practical complex naturalistic task at 6-month follow-up.

Several factors may have contributed to pGMT not replicating improved daily-life $\mathrm{EF}$ as seen in the adult version. First, we have to consider the impact of developmental factors. The young brain has the capacity for more efficient neural restitution, by neural regrowth and anatomical reorganization [71, 72] which may give expectations of a particularly trainable period during adolescence. However, the young brain is also more vulnerable to more severe, diffuse, and enduring deficits after $A B I$ compared to the adult brain [5, 73]. Additionally, the protracted development of EFs and metacognition may have affected metacognitive training effects [74].

When tailoring metacognitive treatments, duration of training is an important factor $[19,75]$ for implementation and automatization of metacognitive strategies [74]. Unlike pBHW, pGMT requires repeated practice of specific techniques. In a meta-analysis, more GMT hours (i.e., higher dose) was associated with greater positive effects on EF [19]. In the present study the participants received $14 \mathrm{~h}$ of hospital training, which might not have been enough time to practice and consequently consolidate and automatize the pGMT strategies to take full advantage of the method in daily life.

Age at the time of training may influence the effects of metacognitive training [74] since previous research has documented greater improvements in late adolescent and adulthood, compared to early adolescence [76, 77]. Although our study was not powered to discover age related differences, the exploratory results did not indicate any difference in effect by age at intervention. Importantly, the referred to studies demonstrating greater improvements in late adolescent included participants in a more acute rehabilitation phase (0-6 months postinsult) than the present study, as well as a more homogeneous sample consisting of only participants with TBI. Larger studies are therefore required to be able to clarify potential differences in the efficacy of pGMT in younger and older adolescents.

Being a metacognitive intervention, pGMT requires self-awareness and reflective skills. Since executive dysfunctions in pABI are associated with lower selfawareness [78], it is likely that for some participants, the high metacognitive demands in pGMT might have surpassed the available resources, thus impeded full comprehension or utilization of the strategies as seen in adults. Even though updating, inhibitory control and the ability to sustain and shift attention are approaching adult capacity in older healthy adolescents, their selfmonitoring and reflective abilities may not fully mature until early adulthood [79].

Further, keeping with recommendations of contextual training and involvement of the adults in the child's life $[30,33]$, which may have resulted in a more robust BHW-comparator than in the adult studies. Powerful non-specific mechanisms common to pGMT and pBHW (e.g., contact with professionals, meeting peers with similar challenges) may have resulted in improvements following both interventions. Indeed, sharing experiences and knowledge replenishment may have enhanced coping strategies or self-efficacy [80]. Further, it has been shown that individual's expectations of efficacy moderate intervention effects [81]. This may, at least partially, explain the improvements in the active control intervention although it was not specifically aimed at EF.

Lastly, assessment of EF is a known challenge [7, 3538 ] and factors related to appropriate EF assessment may have contributed to the small and non-significant differences between interventions. The age-corrected BRIEF scores at baseline were in the normal range [47], which is not in accordance with the executive difficulties described by the parents (and participants) in the screening interview used for inclusion in our study. Rating scales have been criticized for undesirable variability [82] and lack of useful information to assist the best option 
[83]. On the other hand, the screening interview was guided by a research nurse and the respondents were able to more freely describe the real-life EF difficulties compared to the standardized questionnaire. This notion is supported by findings in another RCT $(n=$ 29) evaluating the efficacy of an intervention programme based on social mediation, cooperative learning, and metacognition in pABI [32]. Here, no improvements on the BRIEF were observed when comparing interventions, despite improved metacognitive strategies and improved self-concept assessed with other measures [32], thus questioning whether the BRIEF is sensitive enough to measure change in metacognitive function. Additionally, as parents also received the intervention, it is possible that they were made more aware of EF dysfunction in their child post-intervention in comparison to pre-intervention, potentially reporting less dysfunction at baseline and consequently biasing the estimated effect towards a lesser decrease in dysfunction at follow-up [32].

Investigating secondary intervention effects at the impairment level (performance-based tests), however, pGMT had improved inhibition and executive attention when compared to pBHW. The strong emphasis in pGMT on inhibitory control training (i.e., "stop-andthink") may be related to the enhanced capacity for response inhibition observed in pGMT at 6-month followup (reduced inhibition errors), as pBHW increased in errors in the same period. These results are consistent with GMT findings in adult studies [24], suggesting enhanced bottom-up processing [23]. Further, pGMT displayed improvements in executive attention at both post-intervention assessments (when compared to pBHW), which may support underlying alterations in brain networks linked to attentional control [29]. This finding requires neuroimaging studies to be confirmed. As higher-order EF build on core processes [10], enabled by executive attention [44, 84], these enhancements could potentially improve higher-order EFs (e.g., problem solving). The improvements in inhibition and executive attention may indicate that participants in the pGMT group have initiated a response to the metacognitive intervention, although not fully completed. Future research should investigate the sequencing of potential change in EFs following metacognitive interventions, to test the hypothesis of more sensitive EFs (i.e., inhibition) affected earlier in the course compared to others requiring a more prolonged treatment. The finding of pGMT not improving updating (i.e., digit span) and shifting (i.e., TMT4) more when compared to pBHW are in accordance with findings from adult studies [50].

Despite potential enhancements in the underlying core EF processes, pGMT did not result in improved performance when compared to $\mathrm{pBHW}$ in the complex naturalistic task (CCT). Potential insufficient practice of pGMT strategies and/or developmental factors affecting the capacity of taking full advantage of pGMT may also have affected performance in the CCT. On the other hand, a greater reduction in errors on the CCT after pBHW suggests that psychoeducation delivered to both patients and families is beneficial [85]. Thus, enhanced knowledge of brain injury and (dys)function following pBHW may have enhanced coping strategies and selfefficacy [86].

The ecological validity of performance-based tests has been questioned [79] and consequently accelerated the development of questionnaires such as BRIEF to assess real-life behaviours [79]. However, several factors (such as under/over-reporting, social desirability bias) may also affect the accuracy and validity of questionnaires, as they involve multiple executive and non-executive processes, in addition to contextual influences (e.g., motivation), thus posing major interpretational problems and perhaps not, reflecting EF per se [87]. Questionnaires and performance-based tests correlate relatively poorly [35] and may index different underlying constructs, namely, the efficiency of cognitive abilities and success in goal pursuit [35]. Subsequently, novel ecological methods mimicking everyday impairments are warranted. CCT is a novel multitasking method that, although properties have not yet been fully examined, has demonstrated sensitivity to executive dysfunction in children with TBI [63] and, thus, represented EF assessment in a naturalistic setting. Finally, since executive dysfunction is considered to be a trans-diagnostic symptom [88], we were interested in investigating potential effects across mixed aetiologies. The significance of including a heterogeneous sample is not fully known; nevertheless, we believe it is a potential strength to the study that we tested the intervention in a heterogeneous group initially. Adherence was almost total in both interventions, even though adherence is known to be confounded by fatigue and lack of concentration [78], suggesting that both interventions were perceived as feasible and meaningful.

The study's main limitation is the absence of a waitlist control to account for natural change over time. Previous studies have compared GMT to an active control, but comparing pGMT to pBHW may have masked potential effects of pGMT. The design of keeping nonspecific factors similar in the two interventions (i.e., involvement of parents and teachers, professional attention, group dynamics) may have produced a more robust active control intervention than employed in the adult studies. Although difficult to assess, pre- and postinterventional measures of self-awareness would have been useful to clarify its possible impact on effect. Despite being larger than other paediatric studies in sample size, it is likely that the present study was underpowered 
to detect small differences, especially considering the comprehensive psychoeducation condition employed. The power calculations were performed using the GEC from the BRIEF, rather than the two co-primary endpoints BRI and MI, and were based on a significance level of 0.05 rather than significance levels adjusted for two co-primary outcomes. The sample size was likely to have been too small to detect small differences, if they exist, between the two treatments even if they were potentially clinically relevant. Hence, the results should be regarded as exploratory, and there is a need of future studies with larger sample sizes to confirm our findings. The present study did not explore the potential impact of injury severity on intervention effect. Since injury severity is a well-known predictor of poorer outcome in TBI research [3, 89] and previously has demonstrated impact on interventional effect [90], this should be investigated in future studies. In addition, it is important to consider that the pABI sample in our study was selected based on EF complaints. Thus, our findings may not be generalized to all survivors of pABI.

Future studies should explore subgroup differences in responses to pGMT relating to factors such as age at intervention and severity of insult. Future research should also include objective functional measures (e.g., school performance) and combinations of assessment levels to further clarify the indexed constructs. Finally, future research may consider examining treatment dose (e.g., number of sessions and total treatment time) and the potential effects of "booster sessions" [91].

\section{Conclusion}

To our knowledge, this is one of few high-quality RCTs with blinded assessments on cognitive rehabilitation in the pABI population, addressing several methodological shortcomings from previous studies. Our main findings demonstrated that the metacognitive intervention did not yield additional effectiveness in reducing daily life executive dysfunction compared to pBHW. Moreover, both treatments were associated with distinct effects at different assessment levels of EF. This emphasizes the importance of measuring the various aspects of $E F$ in clinical trials. Considering the high burden of executive dysfunction after pABI, the results point to encouraging clinical implications; both interventions are brief and had high rates of attendance and adherence, indicating high patient acceptance. It is recommended for future clinical trials to explore the sequences of change in EFs, as our secondary findings indicate improvements in inhibition and executive attention after pGMT, suggesting an initiation of response to the metacognitive intervention. Future research will also need to determine possible differences in effects related to treatment duration, developmental factors, and injury characteristics (e.g., severity and aetiology).

\section{Abbreviations}

BRI: Behavioral Regulation Index; BRIEF: Behavior Rating Inventory of Executive Function; CCT: Children's Cooking Task; CPT-III: Conners' Continuous Performance Test, 3ed; CWIT: Color Word Interference Test; DKEFS: Delis-Kaplan Executive Function System; MI: Metacognition Index; pABI: Paediatric acquired brain injury; pBHW: Paediatric Brain Health Workshop; pGMT: Paediatric Goal Management Training; TBI: Traumatic brain injury; TMT: Trail Making Test; WISC-V: Wechsler Intelligence Scale for Children-Fifth edition

\section{Supplementary Information}

The online version contains supplementary material available at https://doi. org/10.1186/s12916-021-02129-8.

Additional file 1:. Semi-structured interview.

Additional file 2:. Stratified analyses Treatment group difference with 95\% confidence intervals.

Additional file 3:. Sensitivity analyses: Treatment group difference with 95\% confidence intervals (Full analysis set/Parent reported).

Additional file 4:. Consort checklist

\section{Acknowledgements}

We would like to thank the participants and their families, Head of the Children's clinic Elisabeth Selvaag and sponsor St. Olavs hospital, Trondheim University Hospital (SOH), in addition to Head of Clinic Terje Rootwelt and Kjersti Ramstad at the Oslo University Hospital (OUH) (Rikshospitalet). Thanks to patient advisers, and the staff at $\mathrm{SOH}$ and $\mathrm{OUH}$, especially our research nurses (Synne Johannesen, Marith Risan, and Line R. Simonsen), occupational therapists (Lene Bobakk and Gøril O. Johansen), neuropsychologists (Helene Eidsmo Barder, Tobias Renzenbrink, and Anne-Britt Skarbø), paediatricians (Selma M. Larsen and Espen Lien), and the test technicians. We also would like to thank Brian Levine, Cathy Catroppa, and Mathilde Chevignard for discussions concerning the study. Paediatrician Bård Forsdahl for help recruiting patients from the University Hospital of North Norway. The Clinical Research Unit Central Norway, Norwegian University of Science and Technology and $\mathrm{SOH}$, with special thanks to Nina Bäcklund for help initiating the project, Anne Caroline Wiik for the Web Clinical Research Form system, and Siril Bakke for help in creating a graphic figure.

\section{Authors' contributions}

AEB contributed to the original study idea, design, protocol, adaptations, and implementation of interventions and is responsible for the statistical analysis plan, statistical analyses, interpretation of data, and drafting of the initial manuscript. TGF contributed to the original study idea, design, protocol, adaptations, and implementation interventions and contributed to the statistical analysis plan. REH contributed to the study design, protocol, adaptations, and implementation of interventions and has contributed to the statistical analysis plan. TBR has alternated the role of principal investigator and has contributed to study design, protocol, funding, and statistical analysis plan. ES is responsible for the statistical analysis plan and for the main statistical outcome analyses. SA contributed to study design and statistical analysis plan. KR has alternated the role of principal investigator, contributed to study design and protocol, and is responsible for the statistical analysis plan and funding and for the main statistical outcome analyses. JS contributed to the original study idea, design, protocol, and statistical analysis plan. He has functioned as a co-principal investigator, contributed to funding, translated GMT to Norwegian, and coordinated the adaptations of the intervention protocols. The authors have read and approved the final manuscript. Finally, all authors have agreed to be accountable for all aspects of the work

\section{Funding}

All phases of this study were supported by The Research Council of Norway [grant number 260680/H10]. Funding organization had no role in the design and conduct of the study; data collection, management, analysis, or 
interpretation; or manuscript review or preparation. Sponsor is a governmental hospital and employer of the research team at St. Olavs hospital, Trondheim University Hospital. St. Olavs sponsor representative did not participate in the work.

\section{Availability of data and materials}

The dataset analysed during the current study are available from the corresponding author on reasonable request.

\section{Declarations}

\section{Consent for publications}

Not applicable.

\section{Ethics approval and consent to participate}

The study was approved by the Regional Committees for Medical and Health Research Ethics, Norway (2017/772/REK), and conducted in accordance to Good Clinical Practice, the Helsinki Declaration, and the standards for Ethical Research Involving Children (ChildWatch International and UNICEF) and reported according to CONSORT criteria [92]. Written informed consent was provided from primary caregivers of participants $(<16$ years) and from participants (> 16 years) and their caregivers.

\section{Competing interests}

The authors declare that they have no competing interests.

\section{Author details}

'Children's Clinic, St. Olavs Hospital, Trondheim University Hospital, Postbox 3250 Torgarden, NO-7006 Trondheim, Norway. ${ }^{2}$ Department of Clinical and Molecular Medicine, Norwegian University of Science and Technology, Trondheim, Norway. ${ }^{3}$ Department of Clinical Neurosciences for Children, Oslo University Hospital, Oslo, Norway. ${ }^{4}$ Department of Public Health and Nursing, Norwegian University of Science and Technology, NTNU, Trondheim, Norway. ${ }^{5}$ Department of Psychology, University of Oslo, Oslo, Norway. ${ }^{6}$ Department of Research, Lovisenberg Diaconal Hospital, Oslo, Norway.

\section{Received: 19 March 2021 Accepted: 14 September 2021}

\section{Published online: 02 November 2021}

\section{References}

1. WHO. World health statistics. Geneva: World Health Organization; 2009.

2. Thurman DJ. The epidemiology of traumatic brain injury in children and youths: a review of research since 1990. J Child Neurol. 2016;31(1):20-7. https://doi.org/10.1177/0883073814544363.

3. Anderson V, Catroppa C, Morse S, Haritou F, Rosenfeld J. Functional plasticity or vulnerability after early brain injury? Pediatrics. 2005;116(6): 1374-82. https://doi.org/10.1542/peds.2004-1728.

4. Muscara F, Catroppa C, Anderson V. The impact of injury severity on executive function 7-10 years following pediatric traumatic brain injury. Dev Neuropsychol. 2008;33(5):623-36. https://doi.org/10.1080/875656408021711 62.

5. Ylvisaker M, Feeney T. Pediatric brain injury: social, behavioral, and communication disability. Phys Med Rehabil Clin N Am. 2007;18(1):13344vii. https://doi.org/10.1016/j.pmr.2006.11.007.

6. Gioia G, Isquith, P., \& Guy, S.: Assessment of executive functions in children with neurological impairment. In: Psychological and developmental assessment: children with disabilities and chronic conditions. Edited by Rosenthal RSS. New York: The Guildford Press; 2001: 317-356.

7. Miyake A, Friedman NP, Emerson MJ, Witzki AH, Howerter A, Wager TD. The unity and diversity of executive functions and their contributions to complex "frontal lobe" tasks: a latent variable analysis. Cogn Psychol. 2000; 41(1):49-100. https://doi.org/10.1006/cogp.1999.0734.

8. Friedman NP, Miyake A. Unity and diversity of executive functions: individual differences as a window on cognitive structure. Cortex. 2017;86: 186-204. https://doi.org/10.1016/j.cortex.2016.04.023.

9. Lehto JE, Juujärvi $P$, Kooistra L, Pulkkinen L. Dimensions of executive functioning: evidence from children. Br J Dev Psychol. 2003;21(1):59-80. https://doi.org/10.1348/026151003321164627.

10. Diamond A. Executive functions. Annu Rev Psychol. 2013;64(1):135-68. https://doi.org/10.1146/annurev-psych-113011-143750.
11. Anderson V, Catroppa C. Recovery of executive skills following paediatric traumatic brain injury (TBI): a 2 year follow-up. Brain Inj. 2005;19(6):459-70. https://doi.org/10.1080/02699050400004823.

12. Catroppa C, Anderson VA, Muscara F, Morse SA, Haritou F, Rosenfeld JV, et al. Educational skills: long-term outcome and predictors following paediatric traumatic brain injury. Neuropsychological Rehabilitation. 2009; 19(5):716-32. https://doi.org/10.1080/09602010902732868.

13. Michaeli O, Kassis I, Shachor-Meyouhas Y, Shahar E, Ravid S. Long-term motor and cognitive outcome of acute encephalitis. Pediatrics. 2014;133(3): e546-52. https://doi.org/10.1542/peds.2013-3010.

14. Stuss DT. Functions of the frontal lobes: relation to executive functions. J Int Neuropsychol Soc. 2011;17(5):759-65. https://doi.org/10.1017/S1355617711 000695

15. Limond J, Leeke R. Practitioner review: cognitive rehabilitation for children with acquired brain injury. J Child Psychol Psychiatry. 2005;46(4):339-52. https://doi.org/10.1111/j.1469-7610.2004.00397.x.

16. Sohlberg MM, Harn B, MacPherson H, Wade SL. A pilot study evaluating attention and strategy training following pediatric traumatic brain injury. Clinical Practice in Pediatric Psychology. 2014;2(3):263-80. https://doi.org/1 $0.1037 /$ cpp0000072.

17. Laatsch L, Dodd J, Brown T, Ciccia A, Connor F, Davis K, et al. Evidencebased systematic review of cognitive rehabilitation, emotional, and family treatment studies for children with acquired brain injury literature: from 2006 to. Neuropsychol Rehabil. 2017;2019(1):1-36. https://doi.org/10.1080/ 09602011.2019 .1678490

18. Cicerone KD, Goldin Y, Ganci K, Rosenbaum A, Wethe JV, Langenbahn DM, et al. Evidence-based cognitive rehabilitation: systematic review of the literature from 2009 through 2014. Arch Phys Med Rehabil. 2019;100(8): 1515-33. https://doi.org/10.1016/j.apmr.2019.02.011.

19. Stamenova $V$, Levine $B$. Effectiveness of goal management training $(R)$ in improving executive functions: a meta-analysis. Neuropsychol Rehabil. 2018:1-31.

20. Levine B, Schweizer TA, O'Connor C, Turner G, Gillingham S, Stuss DT, et al. Rehabilitation of executive functioning in patients with frontal lobe brain damage with goal management training. Front Hum Neurosci. 2011;5:9. https://doi.org/10.3389/fnhum.2011.00009.

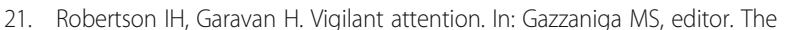
cognitive neurosciences. Cambridge: MIT Press; 2004. p. 631-40.

22. Dams-O'Connor K, Gordon W. Integrating interventions after traumatic brain injury: a synergistic approach to neurorehabilitation. Brain Impairment. 2013; 14(1):51-62. https://doi.org/10.1017/Brlmp.2013.9.

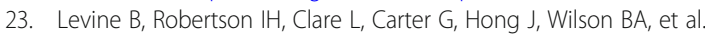
Rehabilitation of executive functioning: an experimental-clinical validation of goal management training. J Int Neuropsychol Soc. 2000;6(3):299-312. https://doi.org/10.1017/S1355617700633052.

24. Tornas S, Lovstad M, Solbakk AK, Schanke AK, Stubberud J. Goal management training combined with external cuing as a means to improve emotional regulation, psychological functioning, and quality of life in patients with acquired brain injury: a randomized controlled trial. Arch Phys Med Rehabil. 2016;97(11):1841-1852.e1843.

25. Spikman JM, Boelen DH, Lamberts KF, Brouwer WH, Fasotti L. Effects of a multifaceted treatment program for executive dysfunction after acquired brain injury on indications of executive functioning in daily life. J Int Neuropsychol Soc. 2010;16(1):118-29. https://doi.org/10.1017/S135561 7709991020.

26. Ryan NP, Anderson V, Godfrey C, Beauchamp MH, Coleman L, Eren S, et al. Predictors of very-long-term sociocognitive function after pediatric traumatic brain injury: evidence for the vulnerability of the immature "social brain". J Neurotrauma. 2014;31(7):649-57. https://doi.org/10.1089/neu.2 013.3153 .

27. Lewis MW, Babbage DR, Leathem JM. Assessing executive performance during cognitive rehabilitation. Neuropsychol Rehabil. 2011;21(2):145-63. https://doi.org/10.1080/09602011.2010.543867.

28. Krasny-Pacini A, Chevignard M, Evans J. Goal management training for rehabilitation of executive functions: a systematic review of effectivness in patients with acquired brain injury. Disabil Rehabil. 2014;36(2):105-16. https://doi.org/10.3109/09638288.2013.777807.

29. Stubberud J, Holthe IL, Lovstad M, Schanke AK, Brandt A, Finnanger T. The feasibility and acceptability of goal management training of executive functions in children with spina bifida and acquired brain injury. Neuropsychological rehabilitation. 2020;31(4):1-20. https://doi.org/10.1080/ 09602011.2020 .1723649 
30. Ylvisaker M, Adelson PD, Braga LW, Burnett SM, Glang A, Feeney T, et al. Rehabilitation and ongoing support after pediatric TBl: twenty years of progress. J Head Trauma Rehabil. 2005;20(1):95-109. https://doi.org/10.1097/ 00001199-200501000-00009.

31. Lindsay S, Hartman LR, Reed N, Gan C, Thomson N, Solomon B. A systematic review of hospital-to-school reintegration interventions for children and youth with acquired brain injury. PLoS One. 2015;10(4): e0124679. https://doi.org/10.1371/journal.pone.0124679.

32. Braga LW, Rossi L, Moretto AL, da Silva JM, Cole M. Empowering preadolescents with $A B I$ through metacognition: preliminary results of a randomized clinical trial. NeuroRehabilitation. 2012;30(3):205-12. https://doi. org/10.3233/NRE-2012-0746.

33. Braga LW, Da Paz AC, Ylvisaker M. Direct clinician-delivered versus indirect family-supported rehabilitation of children with traumatic brain injury: a randomized controlled trial. Brain Inj. 2005;19(10):819-31. https://doi.org/1 0.1080/02699050500110165.

34. Pozzi M, Galbiati S, Locatelli F, Carnovale C, Gentili M, Radice S, et al. Severe acquired brain injury aetiologies, early clinical factors, and rehabilitation outcomes: a retrospective study on pediatric patients in rehabilitation. Brain Inj. 2019;33(12):1522-8. https://doi.org/10.1080/02699052.2019.1658128.

35. Toplak ME, West RF, Stanovich KE. Practitioner review: do performancebased measures and ratings of executive function assess the same construct? J Child Psychol Psychiatry. 2013;54(2):131-43. https://doi.org/1 0.1111/jcpp.12001.

36. Burgess $P$. Theory and methodology in executive function research. In: Rabbitt P, editor. Methodology of frontal executive function. Hove: Psychology Press; 1997. p. 81-116.

37. Miyake A, Friedman NP. The nature and organization of individual differences in executive functions: four general conclusions. Curr Dir Psychol Sci. 2012;21(1):8-14. https://doi.org/10.1177/0963721411429458.

38. Chan RC, Shum D, Toulopoulou T, Chen EY. Assessment of executive functions: review of instruments and identification of critical issues. Arch Clin Neuropsychol. 2008;23(2):201-16. https://doi.org/10.1016/j.acn.2007.08.010.

39. Toplak ME, Connors L, Shuster J, Knezevic B, Parks S. Review of cognitive, cognitive-behavioral, and neural-based interventions for attention-deficit/ hyperactivity disorder (ADHD). Clin Psychol Rev. 2008;28(5):801-23. https:// doi.org/10.1016/j.cpr.2007.10.008.

40. Chevignard MP, Soo C, Galvin J, Catroppa C, Eren S. Ecological assessment of cognitive functions in children with acquired brain injury: a systematic review. Brain Inj. 2012;26(9):1033-57. https://doi.org/10.3109/02699052.2012.666366.

41. WHO. International classification of functioning, disability, and health. Geneva: World Health Organization; 2001.

42. Robertson $\mathbb{H}$, \& Levine, B: Attention and arousal in neurorehabilitation. In: Principles of frontal lobe function. Edited by Knight DTSRT. New York, NY: Oxford University Press; 2013: 742-749.

43. Bilbao A, Kennedy C, Chatterji S, Ustün B, Barquero JLV, Barth JT. The ICF: applications of the WHO model of functioning, disability and health to brain injury rehabilitation. Neurorehabil Neural Repair. 2003;18(3):239-50. https://doi.org/10.3233/NRE-2003-18308.

44. Posner MI, Petersen SE. The attention system of the human brain. Annu Rev Neurosci. 1990;13(1):25-42. https://doi.org/10.1146/annurev.ne.13.030190. 000325.

45. Chevignard MPCC, Galvin J, et al. Development and evaluation of an ecological task to assess executive functioning post childhood TBI: the Children's cooking task. Brain Impairment. 2010;11(2):125-43. https://doi. org/10.1375/brim.11.2.125.

46. Hypher RE, Brandt AE, Risnes K, Ro TB, Skovlund E, Andersson S, et al. Paediatric goal management training in patients with acquired brain injury: study protocol for a randomised controlled trial. BMJ Open. 2019;9(8): e029273. https://doi.org/10.1136/bmjopen-2019-029273.

47. Gioia GA, Isquith PK, Guy SC, Kenworthy L. Behavior rating inventory of executive function: professional manual. Lutz: Psychological Assessment Resources, Inc; 2000.

48. Becker F, Kirmess M, Tornås S, Løvstad M. A description of cognitive rehabilitation at Sunnaas rehabilitation hospital--balancing comprehensive holistic rehabilitation and retraining of specific functional domains. NeuroRehabilitation. 2014;34(1):87-100. https://doi.org/10.3233/NRE-131015.

49. Stubberud J, Langenbahn D, Levine B, Stanghelle J, Schanke AK. Goal management training of executive functions in patients with spina bifida: a randomized controlled trial. J Int Neuropsychol Soc. 2013;19(6):672-85. https://doi.org/10.1017/\$1355617713000209.
50. Tornas S, Lovstad M, Solbakk AK, Evans J, Endestad T, Hol PK, et al. Rehabilitation of executive functions in patients with chronic acquired brain injury with goal management training, external cuing, and emotional regulation: a randomized controlled trial. J Int Neuropsychol Soc. 2016;22(4): 436-52. https://doi.org/10.1017/S1355617715001344.

51. Kabat-Zinn J. Full catastrophe living. New York: Dell Publishing; 1990.

52. Fish J, Evans JJ, Nimmo M, Martin E, Kersel D, Bateman A, et al. Rehabilitation of executive dysfunction following brain injury:"content-free" cueing improves everyday prospective memory performance. Neuropsychologia. 2007;45(6): 1318-30. https://doi.org/10.1016/j.neuropsychologia.2006.09.015.

53. Manly T, Hawkins K, Evans J, Woldt K, Robertson $\mathbb{H}$. Rehabilitation of executive function: facilitation of effective goal management on complex tasks using periodic auditory alerts. Neuropsychologia. 2002;40(3):271-81. https://doi.org/10.1016/S0028-3932(01)00094-X.

54. Varni JW, Limbers CA. The pediatric quality of life inventory: measuring pediatric health-related quality of life from the perspective of children and their parents. Pediatr Clin N Am. 2009;56(4):843-63. https://doi.org/10.1016/j. pcl.2009.05.016.

55. Whyte J, Polansky M, Cavallucci C, Fleming M, Lhulier J, Coslett HB. Inattentive behavior after traumatic brain injury. J Int Neuropsychol Soc. 1996;2(4):274-81. https://doi.org/10.1017/S1355617700001284.

56. Puente AN, Cohen ML, Aita S, Brandt J. Behavioral ratings of executive functioning explain instrumental activities of daily living beyond test scores in Parkinson's disease. Clin Neuropsychol. 2016;30(1):95-106. https://doi. org/10.1080/13854046.2015.1133847.

57. Psychometric properties of the Norwegian version of Behavior Rating Inventory of Executive Function, Parent version (BRIEF-P). PsykTestBarn, 2:6. https://doi.org/10.21337/0037.

58. Donders J, DenBraber D, Vos L. Construct and criterion validity of the behaviour rating inventory of executive function (BRIEF) in children referred for neuropsychological assessment after paediatric traumatic brain injury. J Neuropsychol. 2010;4(Pt 2):197-209. https://doi.org/10.1348/174866409X4 78970 .

59. Wechsler D. Wechsler intelligence scale for children - fifth edition. San Antonio: Psychological Corporation; 2014.

60. Delis DC, Kaplan E, Kramer JH. Delis-Kaplan executive function system: examiners manual: psychological corporation; 2001.

61. Conners $\mathrm{C}$. Conners continuous performance test $3 \mathrm{rd}$ edition ${ }^{\mathrm{TM}}$ (Conners CPT $3^{\text {TM }}$ ) manual. Toronto: Multi-Health Systems Inc; 2014.

62. Chevignard MPSV, Mariller A, et al. Assessment of executive functioning in children after TBI with a naturalistic open-ended task: a pilot study. Dev Neurorehabil. 2009;12(2):76-91. https://doi.org/10.1080/17518420902 777019.

63. Chevignard M, Pillon B, Pradat-Diehl P, Taillefer C, Rousseau S, Le Bras C, et al. An ecological approach to planning dysfunction: script execution. Cortex. 2000;36(5):649-69. https://doi.org/10.1016/S0010-9452(08)70543-4.

64. Moher D, Hopewell S, Schulz KF, Montori V, Gøtzsche PC, Devereaux PJ, et al. CONSORT 2010 explanation and elaboration: updated guidelines for reporting parallel group randomised trials. J Clin Epidemiol. 2010;63(8):e137. https://doi.org/10.1016/j.jclinepi.2010.03.004.

65. Brown H, Prescott R. Applied mixed models in medicine, 3rd edn Chichester: Wiley; 2014. https://doi.org/10.1002/9781118778210.

66. $\mathrm{ICH}$ E9 (R1) addendum on estimands and sensitivity analysis in clinical trials to the guideline on statistical principles for clinical trials. EMA/CHMP/ICH/ 436221/2017 Web site. https://www.ema.europa.eu/documents/scientificguideline/ich-e9-r1-10.addendum-estimands-sensitivity-analysis-clinical-trialsguideline-statistical-principles_en.pdf. Published 17 February 2020. Accessed 12 Feb 2020. In.

67. Bell ML, Floden L, Rabe BA, Hudgens S, Dhillon HM, Bray VJ, et al. Analytical approaches and estimands to take account of missing patient-reported data in longitudinal studies. Patient Relat Outcome Meas. 2019;10:129-40. https:// doi.org/10.2147/PROM.S178963.

68. Hochberg Y. A sharper Bonferroni procedure for multiple tests of significance. Biometrika. 1988;75(4):800-2. https://doi.org/10.1093/biomet/75.4.800.

69. Slomine B, Locascio G. Cognitive rehabilitation for children with acquired brain injury. Dev Disabil Res Rev. 2009;15(2):133-43. https://doi.org/10.1002/ ddrr.56.

70. Baber N. International conference on harmonisation of technical requirements for registration of pharmaceuticals for human use $(\mathrm{ICH}) . \mathrm{Br}$ J Clin Pharmacol. 1994;37(5):401-4. https://doi.org/10.1111/j.1365-2125.1994. tb05705.x. 
71. Giza CC, Prins ML. Is being plastic fantastic? Mechanisms of altered plasticity after developmental traumatic brain injury. Dev Neurosci. 2006;28(4-5):36479. https://doi.org/10.1159/000094163.

72. Kolb B, Pellis S, Robinson TE. Plasticity and functions of the orbital frontal cortex. Brain Cogn. 2004;55(1):104-15. https://doi.org/10.1016/S0278-2626(03 00278-1.

73. Anderson V, Brown S, Newitt H, Hoile H. Long-term outcome from childhood traumatic brain injury: intellectual ability, personality, and quality of life. Neuropsychology. 2011;25(2):176-84. https://doi.org/10.1037/a0021217.

74. Haller EP, Child DA, Walberg HJ. Can comprehension be taught?: a quantitative synthesis of "metacognitive" studies. Educ Res. 1988;17(9):5-8. https://doi.org/10.3102/0013189X017009005

75. Bannert M, Hildebrand M, Mengelkamp C. Effects of a metacognitive support device in learning environments. Comput Hum Behav. 2009;25(4): 829-35. https://doi.org/10.1016/j.chb.2008.07.002.

76. Wade SL, Stancin T, Kirkwood M, Brown TM, McMullen KM, Taylor HG. Counselor-assisted problem solving (CAPS) improves behavioral outcomes in older adolescents with complicated mild to severe TBI. J Head Trauma Rehabil. 2014;29(3):198-207. https://doi.org/10.1097/HTR.0b013e31828f9fe8.

77. Kurowski BG, Wade SL, Kirkwood MW, Brown TM, Stancin T, Taylor HG. Online problem-solving therapy for executive dysfunction after child traumatic brain injury. Pediatrics. 2013;132(1):e158-66. https://doi.org/10.1 542/peds.2012-4040

78. Wolfe KR, Bigler ED, Dennis M, Gerhardt CA, Rubin K, Taylor HG, et al. Selfawareness of peer-rated social attributes in children with traumatic brain injury. J Pediatr Psychol. 2015;40(3):272-84. https://doi.org/10.1093/jpepsy/ jsu060.

79. Anderson P. Assessment and development of executive function (EF) during childhood. Child Neuropsychology. 2002:8(2):71-82. https://doi.org/10.1076/ chin.8.2.71.8724.

80. Wu G, Feder A, Cohen H, Kim JJ, Calderon S, Charney DS, et al. Understanding resilience. Front Behav Neurosci. 2013;7:10. https://doi.org/1 0.3389/fnbeh.2013.00010.

81. Boot WR, Simons DJ, Stothart C, Stutts C. The pervasive problem with placebos in psychology: why active control groups are not sufficient to rule out placebo effects. Perspect Psychol Sci. 2013;8(4):445-54. https://doi.org/1 $0.1177 / 1745691613491271$

82. Campbell JM, \& Hammond, RK.: Best practices in rating scale assessment of children's behavior. In: Best practices in school psychology: data-based and collaborative decision making. edn. Edited by Thomas PLHA. Bethesda, MD: National Association of School Psychologists.; 2014: 287-304.

83. McConaughy SH, \& Ritter, DR.: Best practices in multimethod assessment of emotional and behavioral disorders. . In: Best practices in school psychology: data-based and collaborative decision making. edn. Edited by Thomas PLHA. Bethesda, MD: National Association of School Psychologists.; 2014: 367-389.

84. Posner MI, DiGirolamo GJ. Executive attention: conflict, target detection, and cognitive control. In R. Parasuraman (Ed.), The attentive brain. Cambridge: The MIT Press; 1998. pp. 401-423.

85. Glick ID, Burti L, Okonogi K, Sacks M. Effectiveness in psychiatric care. III: psychoeducation and outcome for patients with major affective disorder and their families. Br J Psychiatry. 1994;164(1):104-6. https://doi.org/10.1192/ bjp.164.1.104.

86. Naismith SL, Diamond K, Carter PE, Norrie LM, Redoblado-Hodge MA, Lewis SJ, et al. Enhancing memory in late-life depression: the effects of a combined psychoeducation and cognitive training program. Am J Geriatr Psychiatry. 2011;19(3):240-8. https://doi.org/10.1097/JGP.0b013e3181dba587.

87. Backeljauw B, Kurowski BG. Interventions for attention problems after pediatric traumatic brain injury: what is the evidence? Pm r. 2014;6(9):81424. https://doi.org/10.1016/j.pmrj.2014.04.004.

88. Snyder HR, Miyake A, Hankin BL. Advancing understanding of executive function impairments and psychopathology: bridging the gap between clinical and cognitive approaches. Front Psychol. 2015;6:328. https://doi. org/10.3389/fpsyg.2015.00328.

89. Catroppa C, Anderson V. Recovery of educational skills following paediatric traumatic brain injury. Pediatr Rehabil. 1999;3(4):167-75. https://doi.org/10.1 080/136384999289432.

90. Resch C, Rosema S, Hurks P, de Kloet A, van Heugten C. Searching for effective components of cognitive rehabilitation for children and adolescents with acquired brain injury: a systematic review. Brain Inj. 2018; 32(6):679-92. https://doi.org/10.1080/02699052.2018.1458335.
91. Tornås S, Løvstad M, Solbakk AK, Schanke AK, Stubberud J. Use it or lose it? A 5-year follow-up study of goal management training in patients with acquired brain injury. J Int Neuropsychol Soc. 2019;25(10):1082-7. https:// doi.org/10.1017/S1355617719000626.

92. Schulz KF, Altman DG, Moher D. CONSORT 2010 statement: updated guidelines for reporting parallel group randomised trials. BMC Med. 2010; 8(1):18. https://doi.org/10.1186/1741-7015-8-18.

\section{Publisher's Note}

Springer Nature remains neutral with regard to jurisdictional claims in published maps and institutional affiliations.
Ready to submit your research? Choose BMC and benefit from:

- fast, convenient online submission

- thorough peer review by experienced researchers in your field

- rapid publication on acceptance

- support for research data, including large and complex data types

- gold Open Access which fosters wider collaboration and increased citations

- maximum visibility for your research: over $100 \mathrm{M}$ website views per year

At BMC, research is always in progress.

Learn more biomedcentral.com/submissions 